\title{
Hyaluronan in cervical epithelia protects against infection-mediated preterm birth
}

\author{
Yucel Akgul, ${ }^{1}$ R. Ann Word, ${ }^{1}$ Laura M. Ensign, ${ }^{2}$ Yu Yamaguchi, ${ }^{3}$ John Lydon, ${ }^{4}$ Justin Hanes, ${ }^{2}$ and Mala Mahendroo ${ }^{1}$ \\ 'Department of Obstetrics and Gynecology and Green Center for Reproductive Biological Sciences, University of Texas Southwestern Medical Center, Dallas, Texas, USA. ${ }^{2}$ Center for Nanomedicine, \\ Johns Hopkins University School of Medicine, Baltimore, Maryland, USA. ${ }^{3}$ Cenetic Disease Program, Sanford Children's Health Research Center, Sanford-Burnham Medical Research Institute, La Jolla, \\ California, USA. ${ }^{4}$ Department of Molecular and Cell Biology, Baylor College of Medicine, Houston, Texas, USA.
}

\begin{abstract}
Increased synthesis of cervical hyaluronan (HA) from early to late pregnancy has long been proposed to play an essential role in disorganization of the collagen-rich extracellular matrix to allow for maximal compliance and dilation of the cervix during the birth process. Here, we show that HA is not essential for increased cervical distensibility during late pregnancy. Rather, cervicovaginal HA plays an unanticipated important role in epithelial barrier protection of the lower reproductive tract. Specifically, HA depletion in the cervix and vagina resulted in inappropriate differentiation of epithelial cells, increased epithelial and mucosal permeability, and strikingly increased preterm birth rates in a mouse model of ascending vaginal infection. Collectively, these findings revealed that although HA is not obligatory for cervical compliance, it is crucial for maintaining an epithelial and mucosal barrier to limit pathogen infiltration of the lower reproductive tract during pregnancy and thereby is protective against infection-mediated preterm birth.
\end{abstract}

\section{Introduction}

Preterm birth (PTB) affects $11.7 \%$ of all pregnancies in the United States. Worldwide, PTB is the primary cause of infant morbidity and mortality in the first postnatal weeks of life and a risk factor for lifelong health problems in those that survive (1). Of the known causes for PTB in women, infection accounts for the largest percentage $(25 \%-40 \%)$ of spontaneous PTB. Ascending microbes from the vagina and cervix are thought to be the primary route for infection. Prophylactic antimicrobial treatment of women has not reduced PTB rates, which suggests that the factors predisposing women to infection-mediated PTB are not confined to the microbiome composition of the reproductive tract $(2,3)$. Parturition, the process of birth, requires timely orchestration of multiple processes that include uterine contractions and remodeling of the cervix from closed and rigid to soft and compliant. Throughout pregnancy, the cervix must protect the fetus from external insults, such as ascending infection from the vagina through the cervix; provide structural integrity for maintenance of pregnancy; and simultaneously undergo progressive remodeling to a distensible organ to allow a safe birth. Regardless of cause, cervical changes precede onset of term and preterm labor in both women and mice $(4,5)$; thus, defining the mechanisms of cervical remodeling in term birth and PTB will allow for improved detection and prevention.

Hyaluronan (HA) is a nonsulfated glycosaminoglycan (GAG) found ubiquitously in the extracellular matrix of all mammalian tissues. During physiologic pregnancy and preterm labor in women and mice, HA levels increase significantly in the cervix (6-10). HA is synthesized by cervical fibroblasts, epithelial cells, and immune cells and released into the extracellular matrix

Conflict of interest: The authors have declared that no conflict of interest exists Submitted: August 27, 2014; Accepted: October 14, 2014.

Reference information: J Clin Invest. 2014;124(12):5481-5489. doi:10.1172/JCI78765.
(ECM) and cervicovaginal mucus. Beginning in early pregnancy, increased synthesis and deposition of collagen with reduced cross-links, along with declined synthesis of specific matricellular proteins, correlates with a significant increase in distensibility by gestation day 12 (d12) in the mouse cervix (11). At the end of pregnancy, increased expression of the HA synthase 2 (Has2) gene, and consequent increases in epithelial and stromal HA, correlate with maximal cervical distensibility, collagen disorganization, and hydration of the cervix (10). In mice, HA at term pregnancy accounts for $1 \%$ of the dry cervical mass, a 5 -fold increase compared with early pregnancy (9). Cervical HA is also increased in mouse models of infection-mediated and non-infection-mediated PTB, although expression is limited to the stromal matrix (9). In addition to its structural role in the ECM, HA has diverse functions - such as cell growth, migration, adhesion, and differentiation that are dependent on HA size and expression of HA-interacting molecules such as CD44, versican, and inter-alpha-trypsin inhibitor (12). The increase in both high- and low-molecular weight HA and cell-specific expression of HA-binding partners in the term cervix suggest that HA may have additional functions in cervical remodeling during parturition that remain to be elucidated (6).

The objectives of our present study were to evaluate the functions of cervical HA during pregnancy and infection-associated PTB using a tissue conditional transgenic mouse model devoid of $\mathrm{HA}$ in reproductive tissues. We tested the hypothesis that the increase in cervical HA at the end of pregnancy leads to maximal cervical distensibility and collagen disorganization that is crucial for a successful parturition, and also evaluated the role of HA in cervical epithelial function in term birth and infection-mediated PTB.

\section{Results}

Conditional HA-depleted mouse models. To evaluate the specific functions of HA in cervical remodeling, we crossed mouse lines 


\section{Table 1. Parturition timing}

$\begin{array}{lc}\text { Group } & \text { On-time incide } \\ \text { WT } & 13 / 13 \\ \text { Has2 KO } & 13 / 13 \\ \text { Has1/3 KO } & 9 / 9 \\ \text { Has1/2/3 KO } & 10 / 10\end{array}$

Timing of parturition was neither premature or delayed by targeted loss of Has2, as pregnant Has2 $\mathrm{KO}$ and Has1/2/3 KO mice delivered on time (i.e., between 6:00 PM on d18 and 12:00 PM on d19), similar to WT and Has1/3 KO females.

with global and tissue-specific KO of the 3 genes that encode HA synthase (Has1, Has2, and Has3) to generate mice in which HA was depleted in the female reproductive tract (see Methods). Targeted mutations in Has 2 result in embryonic lethality (13), whereas mice with global double-KO of Has1 and Has3 (referred to herein as Has $1 / 3 \mathrm{KO})$ develop and grow normally $(14,15)$. To achieve HA depletion in the reproductive tract, we used a $\mathrm{Has}^{\mathrm{Af/f}}$ and heterozygous progesterone receptor $\mathrm{Cre}$ knockin cross ( $\mathrm{Has} 2^{\text {f/ff; }} \mathrm{PR}-\mathrm{Cr}^{+/-}$ mice; referred to herein as Has2 KO) (16). In the Has2 $\mathrm{KO}$ mice, progesterone receptor Cre was expressed throughout the reproductive tract in both epithelia and stroma of the uterus and cervix (Supplemental Figure 1; supplemental material available online with this article; doi:10.1172/JCI78765DS1). Crossing Has1/3 KO mice with Has $2 \mathrm{KO}$ mice resulted in mice with reproductive tract HA depletion (referred to herein as Has1/2/3 KO mice). Cervical HA content, assessed by fluorophore-assisted carbohydrate electrophoresis (FACE), was decreased on average 93\% in Has $2 \mathrm{KO}$ mice and $95 \%$ in Hasl/2/3 KO mice (Figure 1A). Loss of Has 2 gene expression was not compensated with upregulation of Has 1 or Has 3 in cervices of Has2 $\mathrm{KO}$ mice (Figure 1B). Immunofluorescence staining of HA revealed that on d18, the Has $1 / 2 / 3 \mathrm{KO}$ cervical epithelia and mucus were completely devoid of HA, with low levels of HA in stroma (Figure 1, C and D). While immunofluorescence revealed cell localization of insoluble HA that was bound to other ECM structures or to cells, the majority of HA in the cervix was soluble ( $>93 \%$ in WT mice) and could be removed from the sections during the staining procedure (Supplemental Figure 2).

Parturition ratesandcervicalripening. Theincrease of hydrophilic, high-molecular weight HA (500 to $\geq 2,000 \mathrm{kDa}$ ) during cervical ripening has been proposed to play an important role in promoting tissue hydration and collagen matrix disorganization in the cervical stroma, making HA essential for the final changes in tissue compliance required for birth $(6,17)$. Given HA's established function as a structural molecule important in tissue hydration in other ECM-rich tissues, we anticipated that the cervix of HA-depleted mice would fail to undergo reorganization of the collagen matrix, resulting in a parturition defect. Surprisingly, lack of HA did not affect the time of delivery (Table 1), which suggests that cervical HA is not required for normal ripening. Moreover, HA depletion did not affect parity, the increase in hydration during cervical ripening, pubic symphysis elongation, or litter size (Supplemental Table 1).

To determine whether loss of $\mathrm{HA}$ affects cervical ripening, biomechanical properties of cervices from WT and Has $1 / 2 / 3 \mathrm{KO}$ preg- nant mice were assessed late in $\mathrm{d} 18$ and compared with those of WT nonpregnant animals. Tissue stiffness decreased significantly in the d18 WT cervix compared with the nonpregnant WT group (Figure 2A). Interestingly, loss of cervical HA did not prevent a similar decline in tissue stiffness of Hasl/2/3 KO mice in late gestation (Figure 2A and Supplemental Table 2). Although it did not affect cervical function, HA did play a role in space filling, as the spacing between collagen fibers appeared to be decreased in its absence (Figure 2B). Consistent with the biomechanical data, cervical collagen ultrastructure, as determined by measurement of fibril diameter, was similar in d 18 WT and Has $1 / 2 / 3$ KO mice $(P=0.87$; Figure $2 \mathrm{C}$ ). These results collectively suggest that $\mathrm{HA}$ is not obligatory for increased tissue compliance, hydration, and matrix remodeling of the cervix during pregnancy and parturition.

Structure of cervical epithelia. Next, we sought to understand the potential roles of HA in the cervical epithelia, where HA is a component of the pericellular matrix and secreted mucus (Figure 1C). Mucosal epithelia are critical for defense against microorganisms: the mucus forms a physical barrier, and the cells form a junctional barrier and also express pathogen recognition receptors, chemokines, and cytokines that assist in immune surveillance. Staining for alcian blue, Masson trichrome, and the cell-cell adhesion molecule E-cadherin revealed striking disorganization of cervical epithelium in d18 Has1/2/3 KO mice (Figure 3). In particular, the ordered progression of basal epithelia near the basement membrane to polarized mucus-laden epithelial cells facing the lumen in the WT cervix was abnormal in the absence of HA. At the luminal interface, the volume of mucus-containing vacuoles appeared reduced, and the vacuoles were less uniform in shape; moreover, unlike the WT group, epithelial cells with small vacuoles were interspersed with epithelial cells lacking vacuoles, and E-cadherin expression was not uniform across the apical cells. In some regions in the Hasl/2/3 KO mice, the epithelia lacking mucus-laden vacuoles appeared sloughed away from tissue; in other areas, it assumed an altered morphology of elongated squamous epithelia. This pattern was most pronounced in the ectocervix and contiguous vaginal epithelia. Despite the differences in morphology and organization, Has $1 / 2 / 3 \mathrm{KO}$ luminal mucosal epithelia expressed markers of terminal differentiation similar to the WT group, including normal expression of trefoil factor 1 (TFF1), an estrogen-regulated secreted peptide important in restitution and repair of gut epithelia (18). These morphological observations suggested defective proliferation or differentiation of cervical epithelia in the absence of HA. Ki67 and TUNEL staining were indistinguishable between d18 WT and Has $1 / 2 / 3$ KO tissues (Figure 4), which suggests that increased proliferation rate and reduced cell death, respectively, do not account for the aberrant epithelia in the HA-depleted lower reproductive tract.

Compromised cervical epithelial cell differentiation. Epithelial structure was further assessed by immunofluorescent staining of proteins localized to the nonmucosal epithelia (occludin and CD44) in d $18 \mathrm{WT}$ and Has $1 / 2 / 3 \mathrm{KO}$ mice (Figure 5). Expression of CD44, a HA receptor, was strongest in the basal epithelial cells close to the basement membrane and adjacent nonmucosal epithelia, with a gradual decline in expression as cells differentiated into mucosal-laden epithelia. This pattern of expression was similar between WT and Has1/2/3 KO cervical epithelia. Occludin, a 
A

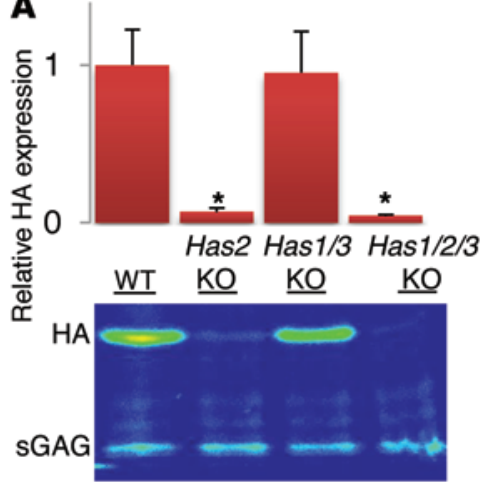

B

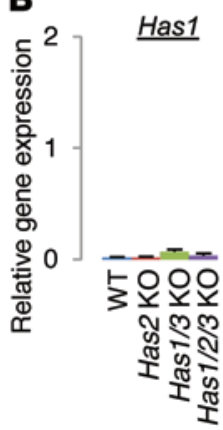

C

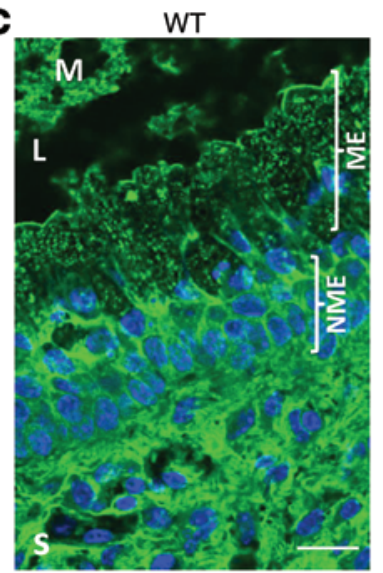

WT
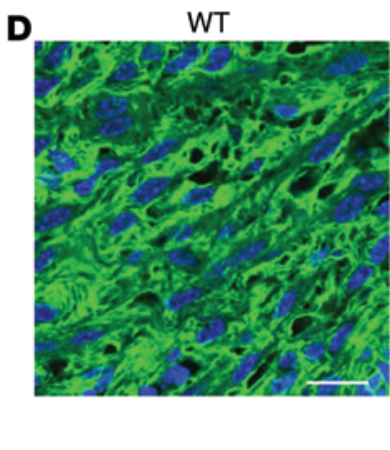

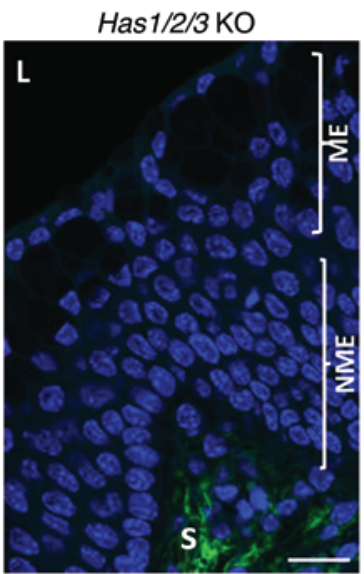

Has $1 / 2 / 3 \mathrm{KO}$

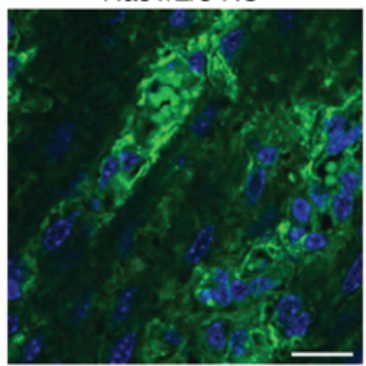

Figure 1. The majority of cervical HA is synthesized by Has2, with $\geq 93 \%$ depletion in Has2 KO and Has1/2/3 KO mice. (A) HA and CS/DS GAGs (SCAG) were quantified by FACE in d18 cervices from WT, Has2 KO, Has1/3 KO, and Has1/2/3 KO mice. $n=3$ per genotype. (B) Decreased Has2 expression in Has2 KO and Has1/2/3 KO cervix. Has1 and Has3, while expressed at low levels in the cervix, did not increase to compensate for Has2 loss. $n=4$ per genotype. (C and D) Visualization of HA (green) and cell nuclei (DAPI; blue) in d18 cervices. While HA was completely depleted from Has $1 / 2 / 3 \mathrm{KO}$ epithelia and mucus (C), low levels of HA were detectable in the stroma of Has2 KO (not shown) and Has1/2/3 KO mice (D), likely derived from progesterone receptor-negative cells. 4 cervices per genotype were evaluated for immunofluorescence. S, stroma; ME, mucosal epithelia (defined as terminally differentiated epithelia with visible mucus-laden vacuoles); NME, nonmucosal epithelia (defined as epithelia with no visible mucus-laden vacuoles); M, mucus; L, cervicovaginal lumen. ${ }^{*} P<0.05,1$-way ANOVA with pairwise multiple comparisons by Tukey test. Scale bars: $20 \mu \mathrm{m}$.

tract led to the hypothesis that loss of HA may result in greater susceptibility to PTB induced by ascending infection of microbes. Live E. coli were inoculated into the vagina of WT and HA-deficient mice on d16. Delivery within 48 hours of inoculation was considered $\mathrm{PTB}$, whereas mice delivering on $\mathrm{d} 19$ were considered term. In the WT group, $50 \%$ of mice exhibited PTB after vaginal inoculation of $10^{7} \mathrm{CFU}$ E. coli, compared with $100 \%$ of the Has $1 / 2 / 3$ KO group $(P=0.02$; Table 2). Similar exposure in Has $2 \mathrm{KO}$ mice resulted in a 92\% PTB rate, although this difference was not statistically significant compared with WT $(P=0.11$; Table 2). The marked difference in susceptibility to ascending infection was confirmed in both Has2 $\mathrm{KO}$ and Has1/2/3 KO mice at a lower E. coli dose of $10^{5} \mathrm{CFU}$ : only $27 \%$ of WT mice exhibited PTB, compared with $92 \%$ of HA-depleted mice (Table 2). Thus, loss of epithelial HA increased susceptibility to infection-induced PTB. This increased susceptibility was dependent on loss of Has2, as PTB rates of Has $1 / 3 \mathrm{KO}$

tight junction protein important in paracellular junctional barrier formation (19), was localized to the same layers of nonmucosal epithelia in Has1/2/3 KO and WT cervix; however, its expression was less apparent in the epithelial cell layers with the strongest CD44 expression (Figure 5A). Regional variations were also observed: occludin expression appeared greater in areas where the luminalfacing epithelia appeared as stratified squamous epithelia with no visible mucus-containing vacuoles (Figure 5B). The apparent disorganization and/or delay in differentiation of the epithelia layers and reduced occludin expression in the absence of HA are suggestive of compromised epithelial function. Expression analysis of genes encoding tight junctions, focal adhesions, gap junctions, adherens junctions, desmosomes, and hemidesmosomes did not reveal significant differences between d18 HA-depleted and WT cervices (Supplemental Figure 3 and Supplemental Table 3). Similarly, expression of genes encoding mucins (Muc2, Muc4, Muc5b, $M u c 13$, and Muc15) were similar in d18 WT and HA-depleted cervices, with the exception of significantly lower $M u c 5 b$ expression in the Has1/2/3 KO group (Supplemental Figure 3).

$P T B$ rates after infection. The aberrant organization of cervical mucosal epithelia in mice lacking HA in the lower reproductive and WT mice were similar (Table 2). The increased sensitivity to infection in HA-depleted mice was accompanied by a more robust inflammatory response, with marked increases in gene expression of both classical and alternatively activated macrophage markers in Hasl/2/3 versus WT cervices (Figure 6). Neither oral administration nor vaginal application of $\mathrm{HA}(200 \mu \mathrm{g} / \mathrm{d}$ on $\mathrm{d} 12-\mathrm{d} 17)$ rescued E. coli-induced PTB in the Has $1 / 2 / 3 \mathrm{KO}$ mice (Supplemental Figure $4 \mathrm{~A})$. The failure to rescue may be due to the fact that neither

\section{Table 2. Infection-induced PTB rates}

\begin{tabular}{lcccccc} 
Group & \multicolumn{2}{c}{ E. coli $\left(\mathbf{1 0} \mathbf{0}^{7}\right.$ CFU) } & \multicolumn{2}{c}{ E. coli $\left(\mathbf{1 0}^{5}\right.$ CFU) } & \multicolumn{2}{c}{ LPS } \\
& PTB rate & $\boldsymbol{n}$ & PTB rate & $\boldsymbol{n}$ & PTB rate & $\boldsymbol{n}$ \\
WT & $50 \%$ & 8 & $27 \%$ & 11 & $29 \%$ & 7 \\
Has1/3 KO & $65 \%$ & 17 & $9 \%$ & 11 & $38 \%$ & 8 \\
Has2 KO & $91 \%$ & 11 & $92 \%{ }^{A}$ & 12 & $67 \%$ & 6 \\
Has1/2/3 KO & $100 \%{ }^{\mathrm{A}}$ & 10 & $92 \%{ }^{\mathrm{A}}$ & 12 & $40 \%$ & 10
\end{tabular}

PTB rates after vaginal exposure to live E. coli $\left(10^{7}\right.$ and $\left.10^{5} \mathrm{CFU}\right)$ or systemic LPS $\left(1 \mu \mathrm{g} /\right.$ mouse i.p.) on d16. ${ }^{A} P<0.05$ vs. WT, Fisher exact test. 
A

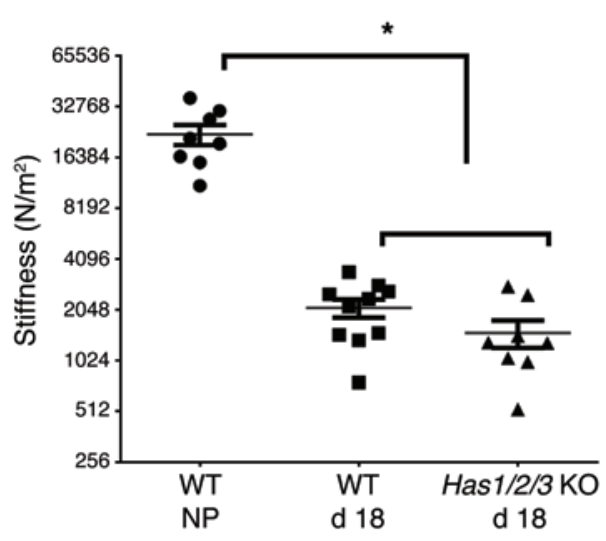

B

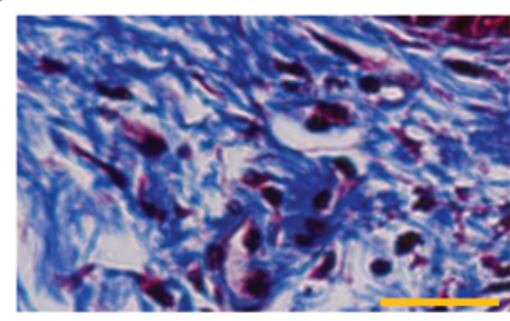

WT
C

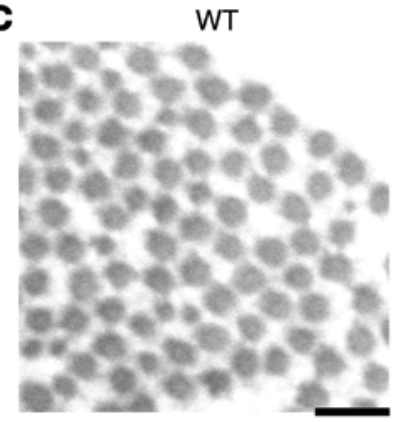

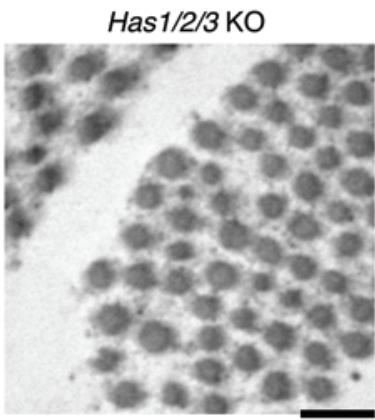

Has 1/2/3 KO

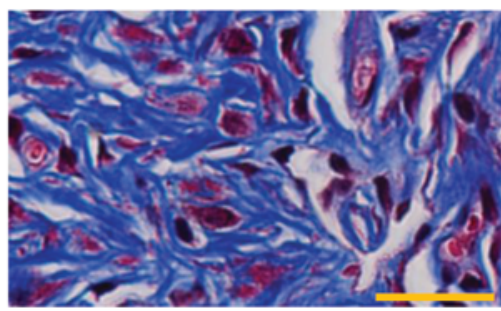

Collagen diameter $(\mathrm{nm})$

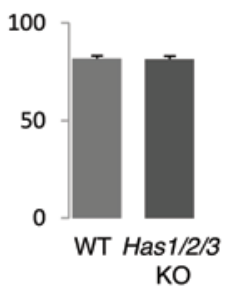

Figure 2. HA is not obligatory for increased tissue compliance during cervical ripening and dilation. (A) Biomechanical assessment of cervical stiffness indicated similar declines in WT and Has1/2/3 KO cervices at term (d18) compared with the nonpregnant (NP) WT cervix. $n=5-8$ per group. (B) Collagen fibers (blue) exhibited similar organization between genotypes, although they appeared more condensed in Has1/2/3 KO than in WT mice. 4 cervices per genotype were evaluated for Masson trichrome stain. (C) TEM of collagen fibrils within the cervical stroma, which were similar in diameter between WT and Has $1 / 2 / 3 \mathrm{KO}$ animals. At least 4 different areas in each of the 3 term cervices per genotype were evaluated for the micrographs. ${ }^{*} P<0.05,1$-way ANOVA with pairwise multiple comparisons by Tukey test. Scale bars: $20 \mu \mathrm{m}$ (B); $200 \mathrm{~nm}$ (C).

route of $\mathrm{HA}$ administration led to significant accumulation of $\mathrm{HA}$ in Has1/2/3 KO cervicovaginal epithelia (Supplemental Figure 4B). In contrast to ascending exposure to infection, PTB rates were similar between WT and Has1/2/3 KO mice given systemic LPS (Table 2). These data indicated that HA-depleted mice were not inherently hypersensitive to systemic infection-induced PTB, but demonstrated increased susceptibility to localized bacteria and ascending infection due to the breach in epithelial integrity. Thus, disrupted barrier function of the cervical epithelia in HA-depleted reproductive tract is the primary defect.

Epithelial and mucosal barrier function. In the next series of experiments, potential mechanisms by which HA depletion affects epithelial and mucosal barrier function were assessed using in vitro permeability assays in a human ectocervical cell line, ECT-1, and in vivo inoculation of microparticles into the mouse vagina. Epithelial permeability assays were carried out in human ECT- 1 cells seeded on inserts with or without siRNA targeting Has3, the primary HA synthase expressed in these cells. In contrast to the pregnant mouse and human cervix, in which HA is synthesized predominantly by HAS2, ECT-1 cells express HAS3. Despite differences in HA synthase isoform expression in the cell line versus cervix, it is well established that HA synthesized from either isozyme is functionally the same (20). The movement of FITC-labeled dextran present in the medium on the inserts to the receiver plate provides a direct measure of cell permeability. siRNA-mediated Has 3 knockdown decreased HA production $60 \%-70 \%$ and increased ECT-1 cell permeability (Figure 7A). However, adding exogenous HA to Has3 siRNA-treated ECT-1 cells did not restore epithelial permeability (Figure 7A). In addition to the epithelial tight junctions, the mucus contributes to the overall barrier function of the epithelia. The integrity of the mucosal barrier was assessed by placement of polyethylene glycol-coated (PEG-coated) polystyrene microparticles (PSPEGs) that were larger in diameter than the mucus pore spacing, but similar in size to E. coli $(1 \mu \mathrm{m})$, into the mouse cervicovaginal canal on d17. Particles densely coated with PEG have mucoinert surfaces, such that coated particles smaller in diameter than the mucus pores were able to penetrate human and mouse cervicovaginal mucus $(21,22)$. However, if the mucus pore spacing is smaller than the particle diameter, even mucoinert particles will become sterically trapped in the mucus mesh (21). At 15 minutes after application of $1 \mu \mathrm{m}$ PSPEGs into the vagina, similar fractions traversed into the uterus in both WT and Has $1 / 2 / 3 \mathrm{KO}$ mice; however, in both genotypes, the majority of the PSPEGs were retained in the cervicovaginal canal (Figure 7B). Higher-magnification images in the ectocervical areas did reveal differences in PSPEG movement: the particles moved deeper into the mucosal layer in Has $1 / 2 / 3 \mathrm{KO}$ versus WT mice, appearing closer to epithelia (Figure 7B). The substantial difference in penetration of mucus by the PSPEGs, which were similar in size to bacteria, suggests that HA within the cervicovaginal mucus plays a role in mucosal barrier protection from pathogens.

\section{Discussion}

Although a substantial number of PTBs in women are associated with ascending infection from the vagina through the cervix, no specific microbe or microbiome profile has been shown to be causative of $\mathrm{PTB}$, and prophylactic antibiotic treatment during or prior to pregnancy does not alter PTB rates $(23,24)$. Our present findings offer a rationale for previous observations and provide new insights into the importance of the cervical epithelia and mucus to form a protective barrier against pathogen invasion, especially 


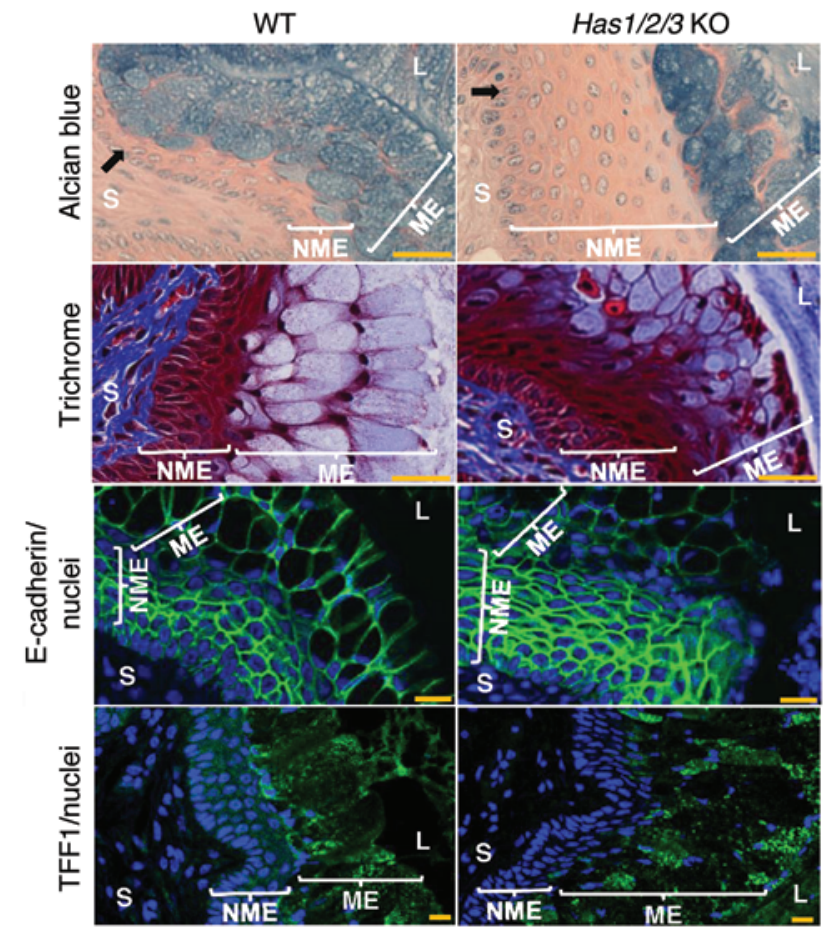

Figure 3. HA depletion results in aberrant organization and differentiation of cervical epithelia. Ectocervical sections from term (late d18) WT and Has $1 / 2 / 3 \mathrm{KO}$ mice were evaluated. Alcian blue stained mucus vacuoles (blue) and nonmucosal cells (pink); arrows denote basal epithelial cells lining the basement membrane. Masson trichrome stained keratin and muscle fibers (red), cytoplasm (pale red), collagen (blue), and nuclei (dark brown/ black). E-cadherin (green) was present on the cell surface of epithelial cells. TFF1 (green) immunofluorescence was localized to the mucus present in vacuoles of luminal epithelia and secreted mucus. In E-cadherin and TFF1 images, nuclei were stained with DAPI (blue). 4 cervices per genotype were evaluated for each stain. Scale bars: $20 \mu \mathrm{m}$.

during extensive remodeling of the cervix during late gestation. HA secreted by the epithelia plays key roles in barrier function. These roles include regulation of epithelial cell differentiation and organization as well as modulation of cervical mucus permeability. The lower expression of the junctional protein occludin observed in $\mathrm{CD}_{4} 4^{+}$epithelial cells of HA-depleted mice (Figure 5, Supplemental Figure 3, and Supplemental Table 3) suggests that HA affects the protein expression and/or subcellular distribution of adhesion/junction molecules, rather than the transcription of these genes. Our observations that HA was necessary for appropriate differentiation of the cervical epithelia, and that its depletion within the cervicovaginal mucosal epithelia increased the vulnerability to microbia-induced PTB, endorse the importance of HA synthesis in the epithelia in maintenance of pregnancy. This func-

Figure 4. Aberrant organization of epithelia in HA-depleted cervices does not result from increased proliferation or apoptosis. Proliferation of cervical basal epithelial cells, as assessed by Ki67 staining (green), indicated similar proliferation rates between WT and Has 1/2/3 KO mice. Apoptosis was assessed by TUNEL (green) counterstained with nuclear marker (red). Arrows denote basal epithelial cells lining the basement membrane. 3 cervices per genotype were evaluated for each stain. Scale bars: $20 \mu \mathrm{m}$. tion expands HA's proposed role in protection of mucosal epithelia, as a recent study reported the importance of HA in breast milk to activate antimicrobial expression in gut mucosal epithelia of the offspring ingesting the milk (25).

The fact that vaginal $\mathrm{HA}$ application from $\mathrm{d} 12$ to $\mathrm{d} 17$ failed to rescue the sensitivity to PTB in Has1/2/3 KO mice, as well as the failed restoration of permeability in HA-treated ECT-1 cells with siRNA-mediated Has3 knockdown, suggest that HA function is more complex than simply improving the barrier properties of the mucus. It is possible that the program of cell differentiation of basal epithelia may require HA exposure specifically to the basal epithelia, which is likely not efficient with vaginal HA application, or that exposure may be required at earlier time points in pregnancy. Our present data indicated that the absence of HA leads to abnormal differentiation and epithelial layer organization and altered mucosal properties that lead to declined barrier function. Barrier function encompasses both the mucosal barrier properties and the paracellular regulation between epithelial cells regulated by junctional proteins. Differences in the biophysical characteristics of cervical mucus from women at high risk of PTB supports the importance of mucosal properties in barrier function (26). Given that transcript expression of mucins, antimicrobial peptides, tight junctions, and adherens junctions were similar between d18 WT and HA-depleted cervices, the function of HA and its binding partners in cervical epithelial function and cell signaling remains to be elucidated. The observed regional and differentiation-specific differences in expression of occludin and E-cadherin protein suggest that epithelial barrier function is compromised, although further studies are required to understand HA's role. Although numerous HA-interacting molecules are expressed in the pregnant cervix to modulate HA function, we propose that the interaction of HA with its receptor, CD44, is likely critical for epithelial differentiation and barrier function. This notion is supported by the fact that squamous epithelial cells, such as keratinocytes, are also dependent on CD44 for normal cellular function. $\mathrm{Cd} 44^{-/}$mice have skin permeability dysfunction, and HA-CD44 interactions are required for mediating intracellular signaling pathways necessary for keratinocyte migration, adhesion, proliferation, and differentiation $(27,28)$.

A second novel finding of the current study is that HA within the stromal ECM was not essential for collagen disorganization and increased hydration of the cervix during the accelerated phase of cervical ripening at the end of pregnancy. During

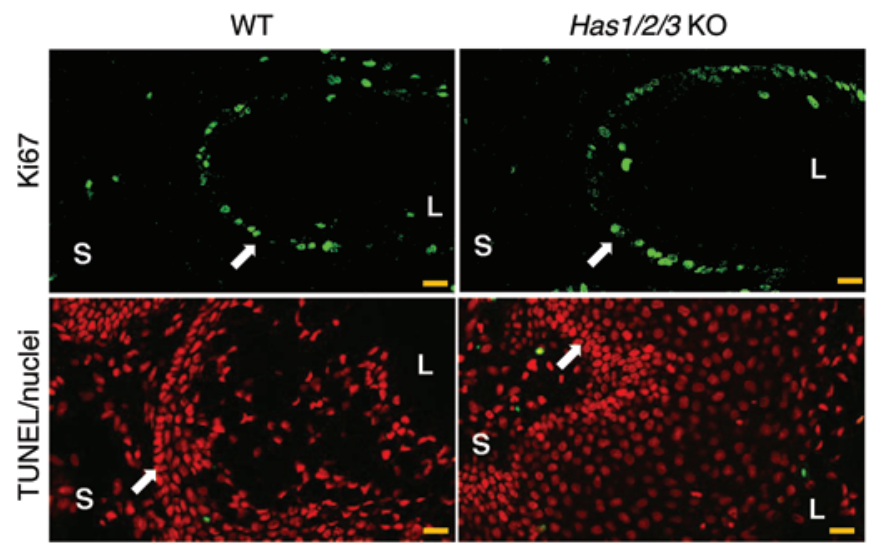



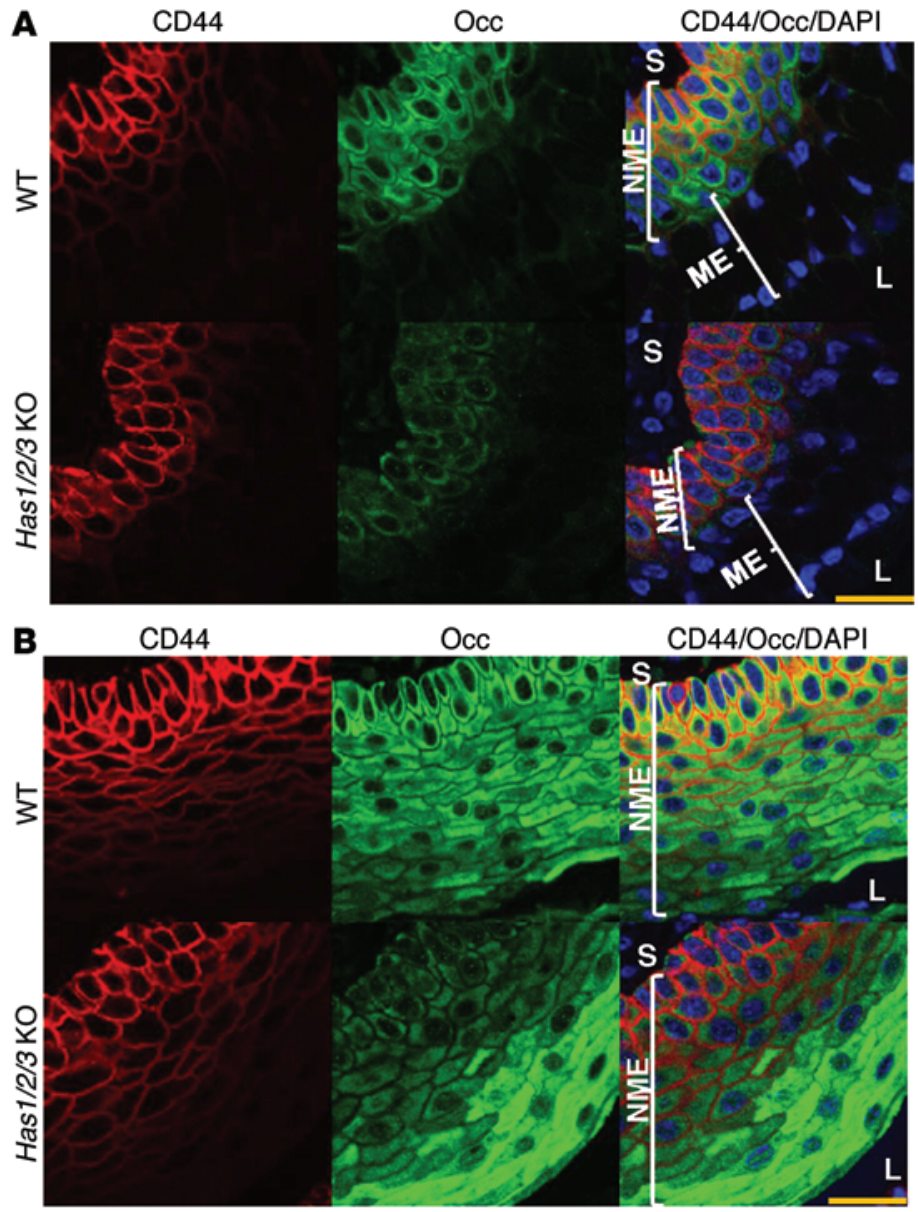

cervical ripening, $\mathrm{HA}$ is produced in a range of sizes from low to high molecular weight. High-molecular weight HA has a wellestablished function as a structural molecule that increases tissue flexibility, elasticity, and/or resilience: it can form crosslinks with the proteoglycan versican to generate structures that are space-filling and increase elasticity of a tissue while maintaining tissue strength. HA has been proposed to be directly linked with ECM disorganization in the term cervix of many species. While collagen bundles were more closely packed in HAdepleted mice, suggesting that HA is involved as a space-filling molecule, collagen fibril size and resulting tissue mechanical strength were not affected (Figure 2). However, our present studies only evaluated cervical stiffness, and it remains possible that HA loss in the cervix may affect other biomechanical parameters, such as recoverability of the tissue (elasticity) or its ability to resist fracturing and breaking (strength). Low-molecular weight HA has proposed immunomodulating functions that include recruitment and activation of leukocytes. HA depletion did not alter the induction of either classical or alternatively activated macrophages; rather, the gene expression response was similar between WT and Has1/2/3 KO mice (Figure 6).

Globally, PTB and stillbirths associated with PTB result in 3.3 million perinatal deaths per year (29). While the direct causes of PTB remain unknown, the primary risk factors for PTB and resulting perinatal death in developing countries are poor nutrition and infection. Despite the magnitude of this health problem, there are
Figure 5. HA depletion diminishes occludin expression in $\mathrm{CD}_{4} 4^{+}$epithelia. (A) CD44 (red) and occludin (Occ; green) revealed decreased occludin expression in the CD44+ epithelial cells of Has $1 / 2 / 3 \mathrm{KO}$ versus WT mice at term (d18). (B) The luminal ectocervical epithelia and contiguous vaginal epithelia displayed reduced volume of mucus-laden vacuoles and increased occludin expression in d18 Has 1/2/3 KO versus WT mice. Nuclei were stained with DAPI (blue). 4 cervices per genotype were evaluated for each stain. Scale bars: $20 \mu \mathrm{m}$.

few effective strategies for diagnosis or prevention of PTB. The unanticipated role of $\mathrm{HA}$ as a protective factor against pathogen-mediated PTB warrants further studies to determine whether reduced cervical HA expression (or increased HA metabolism) may be a susceptibility factor predisposing certain women to infection-associated PTB and whether long-term exogenous HA may reduce risk of infectionmediated PTB. Such findings could readily be translated into cost-effective preventative therapies.

\section{Methods}

Mice. Has1 $1^{-/-}$mice (14) and Has3 $3^{-/-}$mice (15) were generated previously using a strategy that eliminates the catalytic site of each enzyme. Has1 ${ }^{-/-}$and $\mathrm{Has}^{-/-}$mice were intercrossed to generate Has1/3 KO animals, nullizygous for both alleles. Has $2^{f / f l}$ mice (30) were crossed with $\mathrm{PR}-\mathrm{Cre}^{+/-}$mice (16). $\mathrm{Has} 1 / 3 \mathrm{KO}$ and $\mathrm{Has} 2^{f / f f}$; $\mathrm{PR}-\mathrm{Cr}^{+/-}$mice were backcrossed 5 times to generate $\mathrm{Has} 1 / 2 / 3 \mathrm{KO}$ mice. Mice were housed under a 12-hour light/12-hour dark photoperiod at $22^{\circ} \mathrm{C}$ and were of mixed strain (C57BL6/129Sv). Mice in these studies were 2-6 months old and nulliparous. Female mice were housed with males from 4:00 PM to 8:00 AM, then checked for vaginal plugs. The day of plug formation was counted as d0, and birth occurred on d19. Deliveries between 6:00 PM on d18 to 12:00 PM on d19 was considered term (i.e., on time). Tissues from pregnant mice were collected at midday except for d18 samples, which were collected in the evening closer to the time of delivery.

The mouse colony was maintained under clean/sterile conditions, and the health status of mice was assessed on a quarterly basis. Based on tests using sentinel mouse serum, the colony was negative for Mycoplasma pulmonis, epizootic diarrhea of infant mice (EDIM), mouse hepatitis virus (MHV), parvoviruses (MVM and MPV), Sendai virus, Theiler's murine encephalomyelitis virus (TMEV), pinworms, and fur mites.

Tissue collection. Pubic symphysis length was measured using a digital caliper on mice after removing the abdominal wall. Cervices were isolated by dissection at the uterocervical junction, and all vaginal tissue was removed. Wet weights of cervical tissues were collected after tissue dissection. For immunostaining, cervices with vagina and uterine horns were collected for longitudinal sections with the cervical canal throughout each section and in the same longitudinal plane as the uterine horns.

Infection-induced PTB by vaginal E. coli inoculation. Live E. coli (serotype O55; ATCC) (20 $\mu$ l bacteria equal to $\left.10^{5}-10^{7} \mathrm{CFU}\right)$ was inoculated into vagina the morning of $\mathrm{d} 16$, and mice were observed for 48 hours for signs of PTB as described previously (31). To confirm the dose of bacteria given, an aliquot of the leftover bacteria $\left(5 \times 10^{8} \mathrm{CFU} / \mathrm{ml}\right)$ was serially diluted 1:100 to bring down the bacteria concentration to $500 \mathrm{CFU} / \mathrm{ml}$. A 100- $\mu \mathrm{l}$ aliquot was plated under sterile conditions, and 


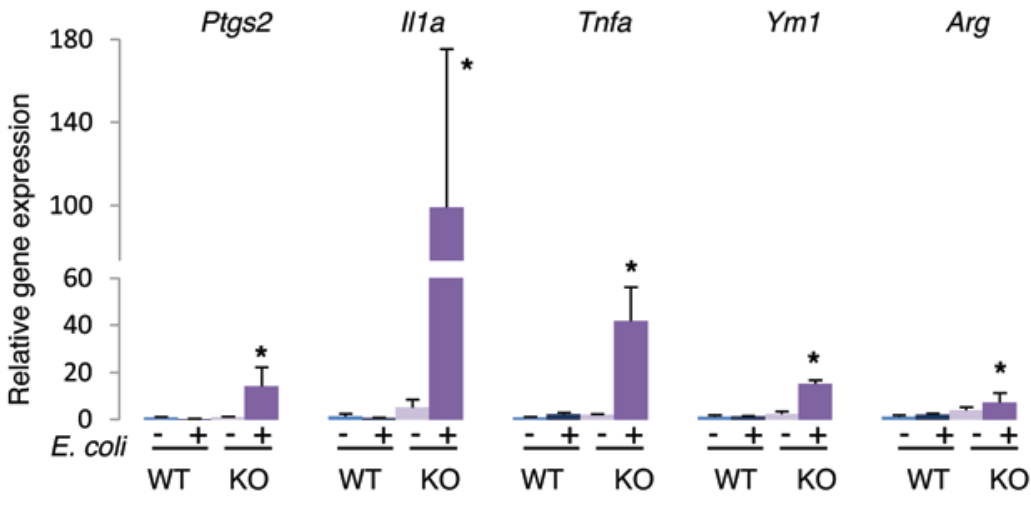

Figure 6. HA depletion compromises epithelial barrier function and increases susceptibility to infection-mediated PTB. Gene expression of classical (Ptgs2, II1a, and Tnfa) and alternative ( $\mathrm{Ym} 1$ and $\mathrm{Arg}$ ) immune response after 24-hour live vaginal $E$. coli exposure ( $\left.10^{5} \mathrm{CFU}\right)$ in WT and Has $1 / 2 / 3 \mathrm{KO}$ (KO) mice. $n=4$ per group. ${ }^{*} P<0.05$ vs. uninfected, 1 -way ANOVA with pairwise multiple comparisons by Tukey test.

colonies were counted after overnight incubation at $37^{\circ} \mathrm{C}$ to confirm the accuracy of the CFU. For mice receiving daily vaginal HA (20 $\mu \mathrm{l}$ HA equal to $100 \mu \mathrm{g}$; intrinsic viscosity, 2.7-3.6 $\mathrm{m}^{3} / \mathrm{kg}$; molecular weight, 1.7-2.4 kDa; catalog no. HA-EP3.0, Stanford Chemicals), the HA was placed in the cervicovaginal canal on the evenings of d12-d17.

Infection-induced PTB by systemic LPS injection. Preterm labor was induced by i.p. injection of $100 \mu \mathrm{l}$ LPS ranging 1-100 $\mu \mathrm{g}$ (strain O:55, Sigma-Aldrich) on d16 in WT and HA-depleted mice. Animals were observed 48 hours after injection for signs of PTB.

FACE. Cervices were collected, and dry weight was measured after lyophilization. Tissues were digested in $100 \mathrm{mM}$ ammonium acetate with $0.0005 \%$ phenol red $(\mathrm{pH} 7.0)$ containing $0.25 \mathrm{mg} / \mathrm{ml}$ proteinase-K (Roche) for 4 hours at $60^{\circ} \mathrm{C}$. Proteinase- $\mathrm{K}$ was inactivated by boiling, and undigested tissues were pelleted by centrifugation. An aliquot of $12.5 \mu \mathrm{l}$ supernatant equal to $0.125 \mathrm{mg}$ dry weight of digested cervix was processed for FACE as described elsewhere (31, 32), with minor modifications. HA and chondroitin/dermatan sulfate (CS/DS) disaccharides were generated by digestion with Hyaluronidase SD $(10 \mathrm{mU})$ at $37^{\circ} \mathrm{C}$ and Chondroitinase $\mathrm{ABC}(10 \mathrm{mU})$ for 3 hours (all GAG digesting enzymes obtained from Associates of Cape Cod Inc.). Digests of HA and CS/DS were fluorescently labeled with 2-aminoacridone (AMAC; Invitrogen). Samples were incubated overnight at $37^{\circ} \mathrm{C}$ with $5 \mu \mathrm{l}$ of $2.5 \mathrm{M} \mathrm{NaCNBH}_{4}$. Following overnight incubation at $37^{\circ} \mathrm{C}, 2.5 \mu \mathrm{l}$ glycerol was added, and 5 - $\mu$ l samples were run on gels. Gels were imaged using a Fuji FLA-5000 phosphoimager. GAG levels were quantified by signal intensity using Fujifilm Multigauge 3.0 software.

Biomechanical testing. Each cervix was suspended between 2 stainless steel wire mounts inserted through the cervical os and attached to a steel rod apparatus with a calibrated mechanical drive and to a force transducer. Tissues were maintained in a physiologic salt solution in water baths at $37^{\circ} \mathrm{C}$ with $95 \% \mathrm{O}_{2}$ and $5 \% \mathrm{CO}_{2}$. After acclimation for 15 minutes, each ring was equilibrated to slack length (ring diameter at resting tone), as measured by the calibrated mechanical drive. Rings were distended in 1-mm increments with 2-minute intervals between each increment to allow stabilization of forces before subsequent distention. This process was continued until failure (breakage) or until plateau of force generation. Stress (newtons per square meter) was were perfused with $4 \%$ paraformaldehyde (Sigma-Aldrich) in PBS, and harvested reproductive tracts were fixed in $4 \%$ paraformaldehyde overnight and kept in PBS until paraffin embedding. Sections (5 $\mu \mathrm{m}$ thick) were deparaffinized and preincubated with 10\% normal donkey serum (Life Technologies) for 20 minutes at room temperature. Subsequently, tissue sections were incubated with $10 \%$ donkey serum containing primary antibody, biotinylated HA binding protein (5 $\mu \mathrm{g} / \mathrm{ml}$; Seikagaku), occludin (1:200 dilution; catalog no. 31721, Abcam), E-cadherin (1:200 dilution; catalog no. 3195, Cell Signaling), CD44 (5 $\mu \mathrm{g} / \mathrm{ml}$; catalog no. MAB2137, Chemicon), TFF1 (1:200 dilution; catalog no. Tff11-A, Alpha Diagnostic), or Ki67 (catalog no. VP-K452, Vector labs) in $10 \%$ normal donkey serum for approximately 16 hours at $4^{\circ} \mathrm{C}$. The slides were washed 3 times with PBS, then incubated with a solution containing fluorescein-tagged streptavidin (1:500 dilution) or Alexa Fluor 488-conjugated secondary antibodies (Ig, heavy and light chains; 1:1,000 dilution; Life Technologies) in $10 \%$ normal donkey serum. This secondary incubation was done for 1 hour at $25^{\circ} \mathrm{C}$. Slides were washed 3 times in PBS, and coverslips were mounted to the slides with Prolong Gold containing DAPI (Life Technologies). Occludin and E-cadherin sections were subject to antigen retrieval with boiling in sodium citrate buffer (10 mM, pH 6) for 30 minutes prior to blocking. TUNEL staining for apoptotic cells was done using paraffin sections ( $5 \mu \mathrm{m}$ thick) according to the protocol supplied with the Promega DeadEnd Fluorometric TUNEL System (Promega). Apoptotic cells were labeled with fluorescein, and sections were counterstained with propidium iodide. Images were obtained using a Leica TCS-SP laser scanning confocal microscope (Leica). Image J software was used to generate individual images. Paraffin processing, embedding, sectioning, and histological staining with alcian blue ( $\mathrm{pH}$ 2.5) and Masson trichrome were performed as described previously (33). For alcian blue staining, tissues were fixed in Carnoy's fixative (60\% ethanol, $30 \%$ chloroform, and $10 \%$ glacial acetic acid) before paraffin embedding to preserve mucus.

Transmission electron microscopy (TEM). Mice were perfused with $1 \%$ glutaraldehyde and $2 \%$ paraformaldehyde fixative in $0.1 \mathrm{M}$ phosphate buffer. Cervical and uterine tissue was removed and fixed in $2.5 \%$ glutaraldehyde in $0.1 \mathrm{M}$ cacodylate buffer containing $0.1 \%$ ruthenium red overnight at $4^{\circ} \mathrm{C}$. Tissue was then cut into $250-\mu \mathrm{m}$ 
A

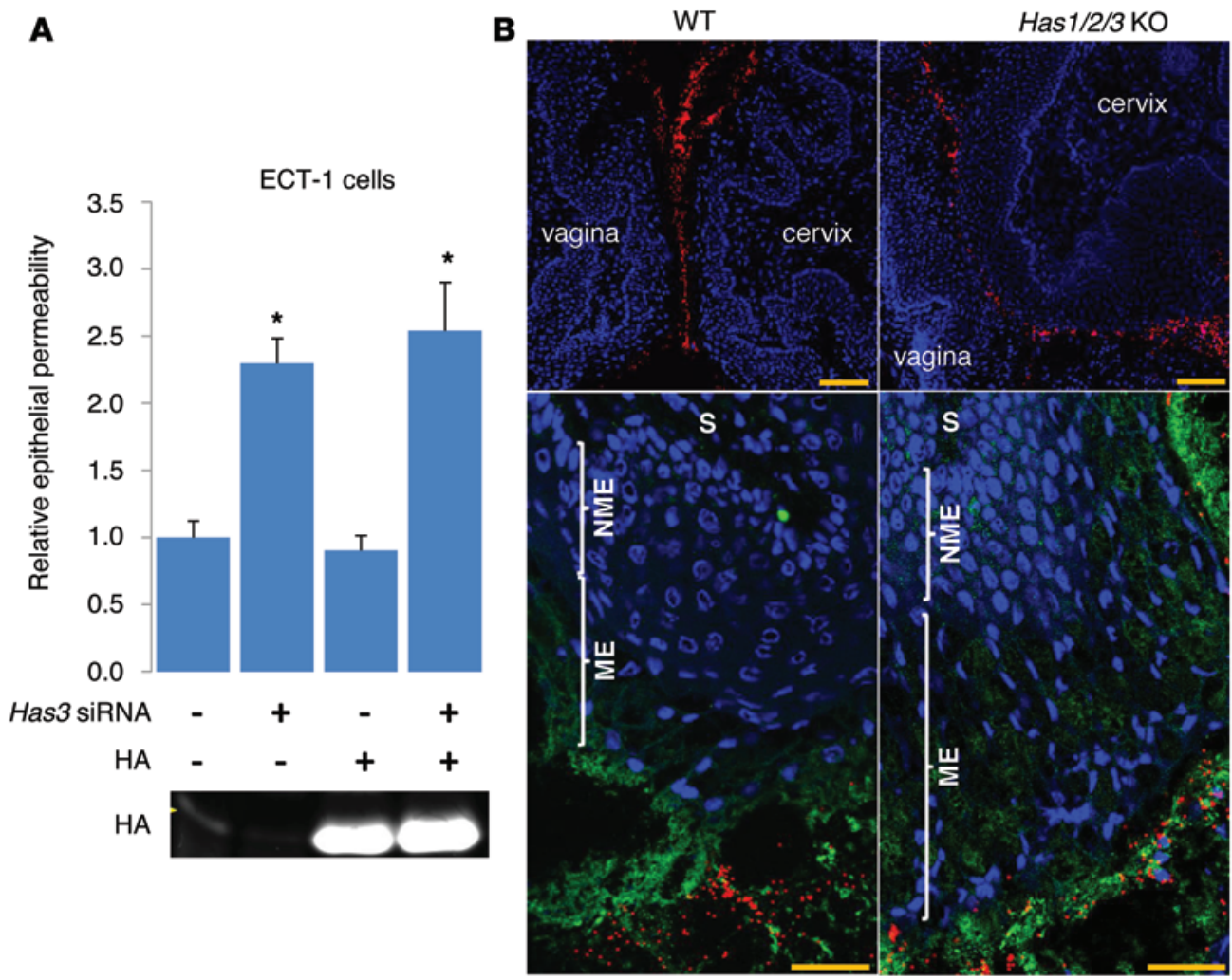

Figure 7. HA depletion compromises epithelial and mucosal barrier functions. (A) siRNA-mediated Has3 silencing increased permeability of confluent ECT-1 cells. However, treatment with exogenous HA did not restore siRNA-treated cells to control levels. FACE analysis confirmed 60\%-70\% HA knockdown in Has3 siRNA-treated groups. (B) Fluorescent PSPEGs (red) penetrated deeper into the mucus layer in Has1/2/3 KO mice than in WT controls. TFF1 staining (green) marks mucus and mucosal epithelia. Nuclei were stained with DAPI (blue). $n=4-5$ per group. ${ }^{*} P<0.05,1$-way ANOVA with pairwise multiple comparisons by Tukey test. Scale bars: $50 \mu \mathrm{m}$.

(Thermo Scientific) premixed with transfection reagent Hyperfect (catalog no. 301704, Qiagen) with or without HA $(1 \mu \mathrm{g} / \mathrm{ml}$; catalog no. HA-EP3.0, Stanford Chemicals) until confluent (about 42 hours) in keratinocyte serum-free medium (KSFM; Life Technologies) supplemented with $50 \mu \mathrm{g} / \mathrm{ml}$ bovine pituitary extract (BPE), 0.1 $\mathrm{ng} / \mathrm{ml}$ epidermal growth factor (EGF), and penicillin-streptomycin at $37^{\circ} \mathrm{C}$ in a $5 \% \mathrm{CO}_{2}$ humidified incubator. The cell culture inserts were transferred to a fresh 24-well plate containing DMEM without phenol. The culture medium was replaced with FITC-labeled dextran diluted in DMEM without phenol and incubated in the dark for 2 hours at room temperature. The amount of FITC-labeled dextran that diffused from inserts through confluent cells into the receiver wells was measured using a microplate reader (TECAN), with $485 \mathrm{~nm}$ excitation wavelength and $535 \mathrm{~nm}$ emission wavelength.

In vivo evaluation of cervicovaginal mucus with fluorescent mucoinert carboxyl microparticles. The integrity of the mucosal barrier was assessed by vaginal adminis-

longitudinal sections using a Vibratome Series 3000 sectioning system (Vibratome Co.). Vaginal and uterine tissue was removed from the cervix, and the cervix was rinsed for 30 minutes with cacodylate buffer. The tissue was postfixed with $1 \%$ osmium in $0.1 \mathrm{M}$ cacodylate buffer containing $0.1 \%$ ruthenium red for 90 minutes. Specimens were dehydrated through a graded series of ethanols $(50 \%$, $70 \%, 95 \%$, and $100 \%$ ) followed by propylene oxide and embedded in Epon (EMbed-812; Electron Microscopy Sciences). Thin sections were stained with uranyl acetate and lead citrate. Sections were viewed by TEM with a Tecnai microscope (FEI Co.), and images were captured with a Morada 11 1-megapixel charge-coupled device camera. Collagen diameter was measured using images taken at $\times 20,500$ magnification using Image J software. Images first underwent threshold optimization prior to measurement of collagen fibril area by the "Analyze Particles" function. Only the circular collagen fibrils were used for the diameter calculation from area excluding diagonal and longitudinal collagen fibrils.

Epithelial permeability assay. The permeability of immortalized ectocervical cells (ECT-1 line; catalog no. CRL-2614, ATCC) was determined by measuring the transepithelial diffusion of FITCtagged dextran by an in vitro Vascular Permeability Assay (catalog no. ECM644, Millipore) as described by the manufacturer. Briefly, ECT-1 cells were plated on cell culture inserts at a concentration of $5 \times 10^{5}$ cells/well and cultured with a pool of nontarget or Has 3 siRNAs tration of PSPEGs. The PEG coating renders the particles mucoinert, allowing for evaluation of cervicovaginal mucus properties as described elsewhere $(21,22)$, with modifications. $7 \mu \mathrm{l}$ of $1-\mu \mathrm{m}$ particles in PBS were inoculated into the vagina on $\mathrm{d} 17$ for 15 minutes, after which the lower reproductive tract - including vagina, cervix, and part of the uterus - was isolated and frozen in Tissue-Tek OCT Compound (Sakura Finetek USA Inc.). Longitudinal sections $(8 \mu \mathrm{m})$ were stained with ProLong Gold antifade reagent with DAPI (Invitrogen) to visualize cell nuclei and retain particle fluorescence. In some experiments, PSPEGs were covisualized with TFF1, a protein secreted into the cervicovaginal mucus. For covisualization, sections were preincubated with $10 \%$ normal donkey serum (Life Technologies) for 20 minutes at room temperature. Tissue sections were then incubated with $10 \%$ donkey serum containing TFF1 antibody (1:200 dilution; catalog no. Tff11-A, Alpha Diagnostics) for approximately 2 hours at room temperature. The slides were washed 3 times with PBS, and then incubated with a solution containing Alexa Fluor 488-conjugated secondary antibodies (Ig, heavy and light chains; 1:1,000 dilution; Life Technologies) in $10 \%$ normal donkey serum. This secondary incubation was done for 1 hour at $25^{\circ} \mathrm{C}$. Slides were washed 3 times in PBS, and coverslips were mounted to the slides in Prolong Gold with DAPI (Life Technologies).

RNA isolation and quantitative real-time PCR. Total RNA was extracted using RNA Stat-60 (Tel-Test) and treated with DNase-I (DNA-Free; Ambion). cDNA synthesis was performed using $1 \mu \mathrm{g}$ total 
RNA/20 $\mu$ l volume iScript Reverse Transcription Supermix (Bio-Rad). Quantitative real-time PCR was performed using SYBR Green and a PRISM 7900HT Sequence Detection System (Applied Biosystems). Aliquots (20 ng) of cDNA were used for each reaction and were run in triplicate. Each gene was normalized to the expression of the housekeeping genes cyclophilin (mouse) or 36B4 (human). Gene expression was calculated according to the $2^{-\Delta \Delta \mathrm{Ct}}$ method, as described by the manufacturer (user bulletin no. 2; Applied Biosystems). The panel of cell junction genes in cervices (Supplemental Table 3) was assayed using the $\mathrm{RT}^{2}$ Profiler PCR Array Mouse Cell Junction Pathway Finder Kit (Qiagen) according to the manufacturer's instructions.

Statistics. Data were analyzed using 1-way ANOVA with pairwise multiple comparisons performed with Tukey test for data normally distributed. Data are displayed as mean \pm SEM. Differences in PTB rates between genotypes (Table 2 and Supplemental Figure 4) were analyzed using Fisher exact test. A $P$ value less than 0.05 was considered statistically significant.

Study approval. All animal studies were conducted in accordance with the standards of humane animal care as described in the $\mathrm{NIH}$ Guide for the Care and Use of Laboratory Animals. The research protocols were approved by the IACUC office at University of Texas Southwestern Medical Center. Because human cells used in this study were an immortalized cell line from normal cervical epithelia purchased from ATCC, human subject approval and informed consent were not required.

\section{Acknowledgments}

We thank F.J. DeMayo (Baylor College of Medicine, Houston, Texas, USA) for providing $\mathrm{PR}-\mathrm{Cr}^{+/-}$mice, J. McDonald (Mayo Clinic Scottsdale, Scottsdale, Arizona, USA) for generation of the $\mathrm{Has3}^{-/-}$mouse, N. Itano and K. Kimata (Aichi Medical University, Aichi, Japan) for generation of the Has1 ${ }^{-/-}$mouse, and E.V. Maytin and V.C. Hascall (Cleveland Clinic, Cleveland, Ohio, USA) for providing Has1/3 KO mice. This work was supported by NIH grant 5-PO1-HD011149.

Address correspondence to: Mala Mahendroo, Department of Obstetrics and Gynecology and Green Center for Reproductive Biological Sciences, University of Texas Southwestern Medical Center, 5323 Harry Hines Blvd., Dallas, Texas 75235-9032, USA. Phone: 214.648.3091; E-mail: mala.mahendroo@utsouthwestern.edu.
1. Martin JA, Hamilton BE, Ventura SJ, Osterman MJ, Mathews TJ. Births: final data for 2011 Natl Vital Stat Rep. 2013;62(1):1-69.

2. Romero R, Mazor M. Infection and preterm labor. Clin Obstet Gynecol. 1988;31(3):553-584.

3. Romero R, Chaiworapongsa T, Espinoza J. Micronutrients and intrauterine infection, preterm birth and the fetal inflammatory response syndrome. J Nutr. 2003;133(5 suppl 2):1668S-1673S.

4. Akgul Y, Mahendroo M. Cervical changes accompanying birth. In: Croy BA, Yamada AT, DeMayo FJ, Adamson SL, eds. The Guide to Investigation of Mouse Pregnancy. Boston, Massachusetts, USA: Academic Press; 2014:391-401.

5. Cunningham F, Leveno K, Bloom S, Spong CY, Dashe J. Williams Obstetrics 24/E. 24th ed. New York, New York, USA: McGraw Hill Professional; 2014.

6. Ruscheinsky M, De la Motte C, Mahendroo M. Hyaluronan and its binding proteins during cervical ripening and parturition: dynamic changes in size, distribution and temporal sequence. Matrix Biol. 2008;27(5):487-497.

7. Osmers R, Rath W, Pflanz MA, Kuhn W, Stuhlsatz HW, Szeverenyi M. Glycosaminoglycans in cervical connective tissue during pregnancy and parturition. Obstet Gynecol. 1993;81(1):88-92.

8. Tateyama N, Asakura H, Takeshita T. Correlation between an absence of cervical gland area on transvaginal sonography and cervical mucus hyaluronic acid levels in women with threatened preterm delivery. J Perinat Med. 2013;41(2):151-157.

9. Akgul Y, Holt R, Mummert M, Word A, Mahendroo M. Dynamic changes in cervical glycosaminoglycan composition during normal pregnancy and preterm birth. Endocrinology. 2012;153(7):3493-3503.

10. Straach KJ, Shelton JM, Richardson JA, Hascall VC, Mahendroo MS. Regulation of hyaluronan expression during cervical ripening. Glycobiology. 2005;15(1):55-65.

11. Akins ML, Luby-Phelps K, Bank RA, Mahendroo
M. Cervical softening during pregnancy: regulated changes in collagen cross-linking and composition of matricellular proteins in the mouse. Biol Reprod. 2011;84(5):1053-1062.

12. Dicker KT, Gurski LA, Pradhan-Bhatt S, Witt RL, Farach-Carson MC, Jia X. Hyaluronan: A simple polysaccharide with diverse biological functions. Acta Biomaterialia. 2014;10(4):1558-1570.

13. Camenisch TD, et al. Disruption of hyaluronan synthase-2 abrogates normal cardiac morphogenesis and hyaluronan-mediated transformation of epithelium to mesenchyme. JClin Invest. 2000;106(3):349-360.

14. Kobayashi N, et al. Hyaluronan deficiency in tumor stroma impairs macrophage trafficking and tumor neovascularization. Cancer Res. 2010;70(18):7073-7083.

15. Bai K-J, et al. The role of hyaluronan synthase 3 in ventilator-induced lung injury. Am J Respir Crit Care Med. 2005;172(1):92-98.

16. Soyal SM, et al. Cre-mediated recombination in cell lineages that express the progesterone receptor. genesis. 2005;41(2):58-66.

17. Timmons B, Akins M, Mahendroo M. Cervical remodeling during pregnancy and parturition. Trends Endocrinol Metab. 2010;21(6):353-361.

18. Taupin D, Podolsky DK. Trefoil factors: initiators of mucosal healing. Nat Rev Mol Cell Biol. 2003;4(9):721-732.

19. Blaskewicz CD, Pudney J, Anderson DJ. Structure and function of intercellular junctions in human cervical and vaginal mucosal epithelia. Biol Reprod. 2011;85(1):97-104.

20. Spicer AP, Tien JY. Hyaluronan and morphogenesis. Birth Defects Res C Embryo Today. 2004;72(1):89-108.

21. Lai SK, et al. Rapid transport of large polymeric nanoparticles in fresh undiluted human mucus. Proc Natl Acad Sci U S A. 2007;104(5):1482-1487.

22. Ensign LM, et al. Mucus-penetrating nanoparticles for vaginal drug delivery protect against herpes simplex virus. Sci Transl Med. 2012;4(138):138ra79.

23. Muglia LJ, Katz M. The enigma of spontaneous preterm birth. N Engl JMed. 2010;362(6):529-535.

24. Iams JD, Romero R, Culhane JF, Goldenberg RL. Primary, secondary, and tertiary interventions to reduce the morbidity and mortality of preterm birth. Lancet. 2008;371(9607):164-175.

25. Hill DR, et al. Human milk hyaluronan enhances innate defense of the intestinal epithelium. J Biol Chem. 2013;288(40):29090-29104.

26. Critchfield AS, et al. Cervical mucus properties stratify risk for preterm birth. PLoS One. 2013;8(8):e69528.

27. Kirschner N, et al. CD44 regulates tight-junction assembly and barrier function. J Invest Dermatol. 2010;131(4):932-943.

28. Bourguignon LY. Matrix hyaluronan-activated CD44 signaling promotes keratinocyte activities and improves abnormal epidermal functions. Am J Pathol. 2014;184(7):1912-1919.

29. Oestergaard MZ, et al. Neonatal mortality levels for 193 countries in 2009 with trends since 1990: a systematic analysis of progress, projections, and priorities. PLoS Med. 2011;8(8):e1001080.

30. Matsumoto K, et al. Conditional inactivation of Has 2 reveals a crucial role for hyaluronan in skeletal growth, patterning, chondrocyte maturation and joint formation in the developing limb. Development. 2009;136(16):2825-2835.

31. Akgul Y, Mahendroo M. Assessment of changes in the peripartum cervix. In: Croy BA, Yamada AT, DeMayo FJ, Adamson SL, eds. The Guide to Investigation of Mouse Pregnancy. Boston, Massachusetts, USA: Academic Press; 2014:723-731.

32. Calabro A, Benavides M, Tammi M, Hascall VC, Midura RJ. Microanalysis of enzyme digests of hyaluronan and chondroitin/dermatan sulfate by fluorophore-assisted carbohydrate electrophoresis (FACE). Glycobiology. 2000;10(3):273-281.

33. Carson FL, Hladik C. Histotechnology: A SelfInstructional Text. Chicago, Illinois, USA: ASCP Press; 2009. 